\title{
Process optimization of reactives systems by partially reduced SQP methods
}

\author{
Marianne von Schwerin a, Olaf Deutschmann ${ }^{\mathrm{b}}$, Volker Schulz ${ }^{\mathrm{c}, *}$ \\ ${ }^{a}$ Siemens AG, Lise-Meitner-Str. 5, D-89081 Ulm, Germany \\ ${ }^{\mathrm{b}}$ Interdisciplinary Center for Scientific Computing, University of Heidelberg, Im Neuenheimer Feld 368, D-69120 Heidelberg, Germany \\ ${ }^{\mathrm{c}}$ Weierstrass Institute for Applied Analysis and Stochastics, Mohrenstrasse 39, D-10117 Berlin, Germany
}

Received 6 April 1998; received in revised form 16 February 2000; accepted 16 February 2000

\begin{abstract}
The increasing demand for optimization in process control of reactive systems necessitates the development of fast and reliable software for the numerical computation of optimal controls taking into account the special structures of the chemical systems under investigation. A recently developed algorithm based on partially reduced SQP methods is used for the computation of optimal controls following the direct approach. In this work, this algorithm is coupled with a package for the simulation of homogeneous reactive systems, which provides the specific routines for the description of the problem at hand. The combined code takes advantage from the fact that the number of influence variables to be determined is rather low compared with the overall number of variables. For example, the computation tool developed is applied to study the homogenous oxidative coupling of methane. (C) 2000 Elsevier Science Ltd. All rights reserved.
\end{abstract}

Keywords: Process optimization; Optimal control; Reactive systems; Partially reduced SQP methods; Methane conversion

\section{Introduction}

Process optimization plays an important role for the efficient use of resources or the minimization of undesired by-products in chemical engineering. In this paper we study in an exemplary manner a homogeneous reactive system, the oxidative coupling of methane, to be optimized with respect to the time-dependent process temperature, as well as to the process duration time. In a similar way we have considered in Schulz and Deutschmann (1999) the efficient formation of ketene from acetic acid. The problem is formulated as an optimal control problem and then solved in a direct approach employing partially reduced SQP methods (Schulz, 1996, 1997). These methods are particularly appropriate for the problem class at hand, since they profit from the fact that, although the total number of variables in the discretized system of equations is large, the number of real process influence variables is small.

* Corresponding author. Tel.: + 49-30-20372553.

E-mail address: schulzv@wias-berlin.de (V. Schulz)
The partial reduction approach is similar to the reduced SQP methods described in Biegler, Nocedal and Schmid (1995); Cervantes and Biegler (1998) and Cervantes and Biegler (2000), but it differs from them in so far as it provides a wider family of methods, of which the latter methods are only one member. Its mathematical convergence theory can be found in Schulz (1996).

As can be imagined from the title, this paper is a report on an interdisciplinary effort on optimization of reactive systems performed by chemical engineers and mathematicians. In so far, it can be considered a promising feasibility study for successful collaboration beyond the narrow circles of each discipline. Therefore, the authors have tried to formulate the contents of each section in such a way that it can be profitably digested by somebody with an education in either discipline.

The paper is organized as follows. In the next section we provide a description of the model of the chemical process to be investigated in this paper. Sections 3 and 4 provide an introduction into the mathematical formulation and practical implementation of partially reduced 
SQP methods for optimal control problems. Section 5 gives numerical results.

The remainder of the current section is devoted to the motivation for the investigation of the particular example.

The conversion of methane, the component of natural gas, has recently received extensive research efforts because of its potential to synthesize useful chemicals and reduce pollutant emissions. Until today, natural gas has mainly been used for combustion process. In future, natural gas could also serve as a clean alternative chemical feed stock. Two essential problems using natural gas are the transportation from the deposits of natural gas to the consumer and the efficient conversion to more valuable chemicals such as ethylene $\left(\mathrm{C}_{2} \mathrm{H}_{4}\right)$.

In principle, natural gas can be transported to the ultimate consumer in pipelines. However, this is often prohibited by geographical or political circumstances. In such cases, this resource can currently not be exploited. The same holds for the natural gas which is set free in the process of producing crude oil. It is usually flared and thus constitutes an inacceptable ecological problem. In recent years, the efforts have been intensified to exploit this kind of natural gas and convert it into liquid hydrocarbons that can be transported more easily.

Several routes have been proposed to convert methane in more useful chemicals which include oxygen in the process: the oxidative coupling of methane by co-feeding of methane and oxygen has been investigated in numerous studies (Lane \& Wolf, 1988). Here, a competition between the coupling products, such as ethylene, and the undesired complete oxidation products, such as $\mathrm{CO}_{2}$ and $\mathrm{H}_{2} \mathrm{O}$, occurs. Therefore, appropriate temperature and residence time have to be chosen to achieve high selectivities of the desired products and a high conversion of methane. Catalytic materials are often used to improve the process. Partial oxidation of methane in monolithic catalysts at very short contact times has recently been shown to offer a promising route to convert natural gas into syngas which can be converted to higher alkanes or methanol (Hickman \& Schmidt, 1993; Deutschmann \& Schmidt, 1998).

These conversion processes can be carried out more efficiently if the conditions in which they operate are not chosen by a trial-and-error treatment, but instead by employing mathematical optimization. So, the most efficient conditions satisfying certain performance criteria can be computed.

In this work the external conditions such as temperature and residence time of the homogeneous oxidative coupling of methane are optimized to achieve maximum concentration of ethylene.

\section{Modeling of the reactive system}

We study a homogeneous chemical reactor with constant pressure and given temperature. Hence, the variables are the species concentrations, the independent variable is the time, and the temperature serves as a time-dependent parameter.

A modern approach to model chemically reactive systems is to use a set of elementary reactions among all chemical species which are involved into the chemical system (Warnatz, Dibble \& Maas, 1996; Warnatz, 1992). This resolution of the reactive system in elementary reactions offers the advantage that the reaction order is directly given by the stoichiometric formulation of the reaction equation and the reaction rates depend only on temperature. Hence, the change of the concentration $c_{i}$ of species $i$ is given by:

$\dot{c}_{i}:=\frac{\mathrm{d} c_{i}}{\mathrm{~d} t}=f_{i}\left(c_{1}, \ldots, c_{N}, T\right)$

$=\sum_{j=1}^{N_{\mathrm{R}}} v_{i j} r_{j} \prod_{k=1}^{N} c_{k}^{v_{k j}}$

with $r_{j}$ as reaction rates, $v_{i j}$ as stoichiometric coefficients, $N_{\mathrm{R}}$ as the number of elementary reactions, $N$ as the number of species. The symbol $T$ denotes the temperature. The rate coefficients $r_{j}$ are given by a modified Arrhenius expression:

$r_{j}=A_{j} T^{\beta_{j}} \exp \left(-\frac{E a_{j}}{R T}\right)$.

For each reaction $j$ the corresponding pre-exponential factor $A_{j}$, the temperature exponent $\beta_{j}$ and the activation energy $E a_{j}$ must be known which is the disadvantage of the detailed chemistry model. Unknown rate constants can be estimated by using methods as discussed, e.g. in Bock (1987); von Schwerin (1997) and Warnatz et al. (1996). However, in the last years, considerable efforts of several groups have been leading to formulation of large reaction schemes including the associated rate expressions (Baulch et al., 1992). Thus, the oxidation of hydrocarbons can be described by a set of elementary reactions.

In the chemical process under consideration we want to maximize the final concentration of a certain species (called here $c_{1}$ ). The temperature profile $T(t)$ in the reactor is set externally, so that the temperature can be employed to control the process in order to achieve the objective. Additionally, the residence time $t_{\text {end }}$ for which the conversion process lasts is free and can be chosen optimally in order to maximize the concentration of the desired species.

For practical reasons, there are often constraints in the design of the chemical reactor which have to be accounted for in the modelling of the process. Hence, the mathematical formulation will include the corre- 
sponding constraints, such as an upper $\left(\mathbf{T}_{u}\right)$ and lower $\left(\mathbf{T}_{l}\right)$ bound for the temperature. Additionally, the optimal residence time $t_{\text {end }}$ must also be restricted by an upper bound $\left(t_{\text {end }_{\max }}\right)$ in order to exclude the case of an infinite process duration. Furthermore, there are positivity requirements and an initial value $\left(c_{i 0}\right)$ has to be specified for the concentration of each species. Hence, the formulation of the optimization problem is as follows:

$\max _{c, \mathbf{T}, t_{\text {end }}} c_{1}\left(t_{\text {end }}\right)$

subject to

$0 \leq t_{\text {end }} \leq t_{\text {end }_{\text {max }}}$

$\mathbf{T}_{1} \leq \mathbf{T}(t) \leq \mathbf{T}_{\mathbf{u}}$

$c_{i}(t) \geq 0, \quad i=1, \ldots, N$

and the dynamics

$\dot{c}_{i}=\sum_{j=1}^{N_{\mathrm{R}}} v_{i j} \mathrm{~A}_{j} T^{\beta_{j}} \exp \left(\frac{\mathrm{E} a_{j}}{\mathrm{RT}}\right) \prod_{k=1}^{N} c_{k}^{v_{k j}}$,

$c_{i}\left(t_{0}\right)=c_{i 0}, \quad i=1, \ldots, N$.

The homogeneous oxidative coupling of methane to higher hydrocarbons such as ethylene $\left(\mathrm{C}_{2} \mathrm{H}_{4}\right)$ and acetylene $\left(\mathrm{C}_{2} \mathrm{H}_{2}\right)$ is studied by cofeeding methane and oxygen at methane rich conditions and temperatures between 800 and $1600 \mathrm{~K}$. For these temperatures a strong competition occurs between complete and partial oxidation of methane as well as conversion to higher hydrocarbons. The first reaction path $\left(\mathrm{CH}_{4}+2 \mathrm{O}_{2} \rightarrow\right.$ $\mathrm{CO}_{2}+2 \mathrm{H}_{2} \mathrm{O}$ ), which leads to undesired products such as $\mathrm{CO}_{2}$ and $\mathrm{H}_{2} \mathrm{O}$, is favored at higher temperatures and is extremely exothermic leading to a further temperature increase (combustion). The partial oxidation of methane $\left(\mathrm{CH}_{4}+1 / 2 \mathrm{O}_{2} \rightarrow \mathrm{CO}+2 \mathrm{H}_{2}\right)$ is more favored in methane rich mixtures. The formed syngas can be extracted from the mixture and converted into higher hydrocarbons and methanol. But here, we are interested in the formation of higher hydrocarbons from methane by a one-step process: $\left(2 \mathrm{CH}_{4}+1 / 2 \mathrm{O}_{2} \rightarrow\right.$ $\mathrm{C}_{2} \mathrm{H}_{6}+2 \mathrm{H}_{2} \mathrm{O}$ and $\mathrm{CH}_{4}+\mathrm{O}_{2} \rightarrow \mathrm{CO}_{2}+\mathrm{C}_{2} \mathrm{H}_{4}+2 \mathrm{H} 2 \mathrm{O}$ ). The temperature is the crucial parameter in this reaction system because it must be high enough to achieve a decent amount of conversion. However on the other side, a higher temperature favors the formation of $\mathrm{CO}$ and $\mathrm{CO}_{2}$. Hence, the problem is to find a proper temperature profile which ignites the global reaction (formation of radicals) while keeping $\mathrm{CO}$ and $\mathrm{CO}_{2}$ formation as low as possible.

It is obvious, that the complexity of the oxidative coupling of methane can only be described satisfactorily by a detailed chemistry model. Therefore, a set of $618\left(=N_{\mathrm{R}}\right)$ elementary reactions among $53(=N)$ chemical species is used. This reaction mechanism includes hydrocarbons containing up to four carbon atoms, and it was tested by numerous simulations of combustion systems and continuous stirred tank reactors (Nehse, 1999). Ethylene $\left(\mathrm{C}_{2} \mathrm{H}_{4}\right)$ is chosen as the higher hydrocarbon species to be maximized. The temperature bounds are $T_{1}=800 \mathrm{~K}$ and $\mathrm{T}_{\mathrm{u}}=1600 \mathrm{~K}$ and the final residence time of the species in the reactor should not exceed $t_{\text {end }_{\max }}=1 \mathrm{~s}$ to guarantee a high enough throughput.

\section{Direct PRSQP methods for optimal control problems}

The aim of this section is to provide a brief description of the mathematical background of the code OCPRSQP, which is used for the core numerical computations in this paper. The problems formulated in the previous section can be summarized under the following general problem class for optimal control problems in differential algebraic equations (DAE).

For $t \in\left[0, t_{f}\right]$, we consider

$\min \phi\left(y\left(t_{f}\right), z\left(t_{f}\right), t_{f}\right)$

objective functional

s.t. $\dot{y}(t)=f(y(t), z(t), u(t))$

$0=g(y(t), z(t), u(t))$

$0=r\left(y(0), z(0), y\left(t_{f}\right), z\left(t_{f}\right)\right)$

model DAE of index 1

$u^{\min } \leq u(t) \leq u^{\max }$

boundary conditions

$s^{\min } \leq s\left(y(t), z(t), u(t) \leq s^{\max } \quad\right.$ state constraints

for the differential variables $y \in \mathbb{R}^{n_{y}}$ the algebraic variables $z \in \mathbb{R}^{n_{z}}$ and the control variables $u \in \mathbb{R}^{n_{u}}$. The inequalities are to be interpreted component-wise. Here we assume that the differential-algebraic equation (DAE), describing the dynamics of the reactive processes, is of index at most 1. For ease of presentation we assume that the functions involved in the formulation posses sufficiently many derivatives. For the treatment of optimization problems in DAE with discontinuities see, e.g. von Schwerin, Winckler and Schulz (1996). Although the actual DAE model of the reactive processes considered in this paper is of index 0 (i.e. pure ordinary differential equations, ODE), we give this presentation in this slightly more general setting because that is the complete problem class which the code OCPRSQP is made for. For the numerical treatment of higher index DAE boundary value problems, leading to natural invariants, see Schulz, Bock and Steinbach (1998).

In principle, there are two ways to treat optimal control problems like the above. One is to use Pontryagin's minimum principle to formulate necessary conditions for the solution of the optimal control problem in the form of a boundary value problem with jumps and switches. This is called the indirect ap- 
proach. It has the potential of computing the exact solution up to the discretization error of the resulting DAE, but it requires good initial guesses for the adjoint variables and for the bang-(off-) bang switching structure. Another possibility is to use the so called direct approach, which is based on an a priori discretization of the states. Direct methods with a multiple-shooting discretization and (full) SQP methods for the solution of the resulting large finite dimensional optimization problem have been discussed in Bock and Plitt (1984) or in the more recent version Leineweber, Bock, Schlöder, Gallitzendörfer, Schäfer and Jansohn (1997). Here we use a collocation discretization and combine it with a new reduced SQP type method, thus profiting from the fact that the number of control variables is small compared to the number of all variables. This approach is described in more detail in the following.

For the control discretization, we choose a grid

$\pi: t_{0}=\tau_{1}<\ldots<\tau_{m}=t_{f}$,

where the control functions are specified piecewise via free control parameters $u_{j}$ and fixed base functions $\chi_{j}$ with local support, $u(t)=\chi_{j}\left(t, u_{j}\right)$ on $\left[\tau_{j}, \tau_{j+1}\right]$. In cases, where $t_{f}$ is left free for optimization, we apply the usual problem reformulation on a fixed time interval with a corresponding time-transformation parameter in the right hand side of the ODE. Thus $\mathrm{u}$ is restricted to some finite-dimensional space of admissible controls independently on each subinterval. For the sake of notational simplicity, in the following we always replace $u(t)$ on $\left[\tau_{j}, \tau_{j+1}\right]$ by $u_{j}$.

For the state discretization we choose a possibly finer sub-grid which for ease of presentation we let coincide with Eq. (3.2) here. Following the lines of Ascher and Spiteri (1994), the differential variables are discretized in the interval $\left[\tau_{j}, \tau_{j+1}\right]$ by the polynomial of order $k+1$

$y^{\pi}(t)=y_{j}+h_{j} \sum_{l=1}^{k} x_{j l} \psi_{l}\left(\frac{t-r_{j}}{h_{j}}\right) \forall t \in\left[\tau_{j}, \tau_{j+1}\right], \quad$ where

$y_{j}:=y^{\pi}\left(\tau_{j}\right) \in \mathbb{R}^{n_{y}}, \quad x_{j l}:=\dot{y}^{\pi}\left(\tau_{j}+\rho_{l} h_{j}\right) \in \mathbb{R}^{n_{y}}$,

$h_{j}:=\tau_{j+1}-\tau_{j}$,

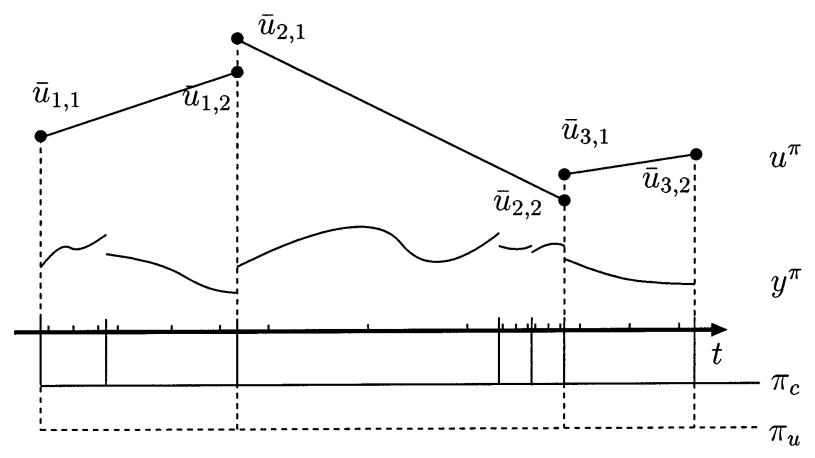

Fig. 1. Discretization of the optimal control problem. $\psi_{l} \in \mathbb{P}_{k+1}[0,1]: \psi_{l}(0)=0, \quad \dot{\psi} l\left(\rho_{i}\right)= \begin{cases}1, & \text { if } l=i, \\ 0, & \text { else }\end{cases}$

The symbol $\mathbb{P}_{k+1}[0,1]$ denotes the space of polynomials of order $k+1$ in the interval $[0,1]$. Here, we have chosen the so-called Runge-Kutta representation of the collocation polynomials. The discretized differential variables, $y^{\pi}(t)$, are required to satisfy the system of differential-algebraic equations in Eq. (3.1) at the collocation points $t_{j l}:=\tau_{j}+\rho_{l}\left(\tau_{j+1}-\tau_{j}\right), \quad l=1, \ldots, k$, where the $k$ numbers $0 \leq \rho_{1}<\ldots \rho_{k} \leq 1$ determine the convergence and stability properties of the collocation discretization. Additionally, they have to be continuous at the grid nodes $\tau_{j}$.

The algebraic variables are discretized by the vectors

$z_{j l} \in \mathbb{R}^{n_{z}}, \quad l=1, \ldots, k, \quad j=1, \ldots, m-1$

representing the solution values $z\left(t_{j l}\right)$ at the collocation points. A polynomial interpolation of $\left\{z_{j l}, \ldots, z_{j k}\right\}$ yields an approximation $z^{\pi}(t)$ in the whole interval $\left[\tau_{j}, \tau_{j}+1\right]$.

Thus the collocation discretization is characterized by the collocated DAE

$x_{j l}-f\left(y_{j}+h_{j} \sum_{i=1}^{k} x_{j i} \psi_{l}\left(\rho_{i}\right), z_{j l}, u\left(t_{j l}\right)\right)=0, \quad l=1, \ldots, k$

$g\left(y_{j}+h_{j} \sum_{i=1}^{k} x_{j i} \psi_{l}\left(\rho_{i}\right), z_{j l}, u\left(t_{j l}\right)\right)=0, \quad j=1, \ldots, m-1$

and the continuity conditions

$y_{j}+h_{j} \sum_{i=1}^{k} x_{j i} \psi_{l}(1)-y_{j+l}=0, \quad j=1, \ldots, m-1$.

Fig. 1 illustrates the discretization described above. The control discretization in this figure is chosen to be piecewise linear. The control mesh, $\pi_{\mathrm{u}}$, and the collocation mesh, $\pi_{\mathrm{c}}$, are shown. The tiny ticks above the $t$-axis indicate the positions of three Gaussian collocation points in each collocation interval.

Ultimately, the direct approach results in a large finite dimensional nonlinear constrained optimization problem of the general form.

$\min \psi(y, u)$

s.t. $c_{1}(y, u)=0, \quad C_{1 y}$ nonsingular,

$c_{2}(y, u)=0$,

$c_{3}(y, u) \geq 0$,

where $y$ denotes the discretized differential and algebraic variables and $u$ the discretized control variables. The constraint $c_{1}$ abbreviates the discretization equations (Eqs. 3.4-3.6) and $c_{2}, c_{3}$ are additional constraints for the states and the controls. In Eq. (3.7) and in the remainder of the paper, capital $C$ symbols with addi- 
tional indices mean derivatives of $\mathrm{c}$ with respect to the variable in the index.

Nonlinear optimization problems of the form Eq. (3.7) without the constraints $c_{2}$ and $c_{3}$ are efficiently treated by reduced SQP methods in the separability framework (Gabay, 1982; Kupfer \& Sachs, 1993), especially if the number of $y$-variables is much larger than the number of the $u$-variables — as is typically the case for chemical process control problems. The idea of reduced SQP methods in contrast to usual SQP methods is to use only an approximation of the projected Hessian of the Lagrangian onto the kernel of the linearized constraint $c_{1}$ instead of an approximation of the full Hessian of the Lagrangian. In order to apply these methods, one must have a global parameterization of the kernel of all active constraints. In the presence of additional equality, $c_{2}$ and in particular of inequality constraints, $c_{3}$, this global parameterization is hard to determine, possibly resulting in instabilities (Gurwitz \& Overton, 1989).

Partially reduced SQP methods (PRSQP) as established in Schulz (1996) overcome these difficulties by combining the advantage of reduced SQP methods small quadratic subproblems - with the advantage of full SQP methods - convenient treatment of inequality constraints. The basic idea of these methods is to formulate the reduced SQP method only with respect to the constraints which allow a straight forward parameterization $\left(c_{1}\right)$, and to treat the remaining constraints $\left(c_{2}, c_{3}\right)$ in the same way as usual SQP methods do, but reduced onto the kernel of the above constraints.

We define

$T_{1}:=\left[\begin{array}{c}-C_{1 y}^{-1} C_{1 u} \\ I\end{array}\right]$

as the basis of the kernel of $C_{1}=\left[C_{1 y} C_{1 u}\right]$.

The Lagrangian of the optimization problem Eq. (3.7) is

$\mathscr{L}=\psi(y, u)-c_{1}(y, u)^{\top} \lambda_{1}-c_{2}(y, u)^{\top} \lambda_{2}-c_{3}(y, u)^{\top} \lambda_{3}$.

A generic PRSQP method is formulated in the following algorithm (additional indices $k$ mean evaluation at the $k$ th iterate):

1. Start at $y_{0}, u_{0}, k:=0$

2. compute the reduced gradient $\gamma_{1, k}:=$ $T_{1, k}^{\top} \nabla \psi_{k}$,determine some appproximation $B_{k}$ of $T_{1, k}^{\top} \frac{\partial^{2}}{\partial^{2}} \mathscr{L}_{k} T_{1, k}$

3. compute the range space solution of the linearized constraint $c_{1}: \Delta y_{k}^{\mathscr{R}}=-C_{1 y, k}^{-1} c_{1, k}$

4. solve the quadratic program

$\min \Delta u_{k}^{\top} B_{k} \Delta_{u k}+\gamma_{1, k}^{\top} \Delta u_{k}$

s.t. $\quad C_{2, k}^{\Delta u_{k}} T_{1, k} \Delta u_{k}+c_{2, k}=-C_{2, k} \Delta y_{k}^{\mathscr{R}}$
$C_{3, k} T_{1, k} \Delta u_{k}+c_{3, k} \geq-C_{2, k} \Delta y_{k}^{\mathscr{R}}$

in order to obtain $\Delta u_{k}$ and adjoint variables and $\lambda_{3, k}$

5. determine step $\Delta y_{k+1}:=\Delta y_{k}^{\mathscr{R}}+T_{1, k} \Delta u_{k}$

6. perform step $y_{k+1}:=y_{k}+\Delta y_{k}$

7. $k:=k+1$, go to (1) until convergence

If the approximation of the reduced Hessian $B_{k}$ is performed in a sufficiently accurate way, e.g. by the BFGS update formula, local 2-step-superlinear convergence can be shown. In order to define these update formulas, one must use the difference in the reduced gradient of the Lagrangian.

$$
\begin{aligned}
q_{k}:= & \bar{\gamma}_{k}-\gamma_{k}-\left[\bar{T}_{1, k}^{\top} \bar{C}_{2, k}^{\top}-T_{1, k}^{\top} C_{2, k}^{\top}\right] \lambda_{2, k} \\
& -\left[\bar{T}_{1, k}^{\top} \bar{C}_{3, k}^{\top}-T_{1, k}^{\top} C_{3, k}^{\top}\right] \lambda_{3, k},
\end{aligned}
$$

where a bar over a symbol means evaluation at an intermediate point $\left(\bar{y}_{k}, \bar{u}_{k}\right)$, and the difference $p_{k}:=$ $\left(\bar{y}_{k}, \bar{u}_{k}\right)-\left(y_{k}, u_{k}\right)$.. These two differences $\left(q_{k}\right.$ and $\left.p_{k}\right)$ are used to impose the secant condition $B_{k} p_{k}=q_{k}$, which is at the core of this type of update formula. The intermediate point may be chosen to be $\left(y_{k+1}, u_{k+1}\right)$, which defines an update strategy in the spirit of Nocedal and Overton (1985), or $\left(y_{k}, u_{k}\right)+\left(T_{1, k} \Delta u_{k}, \Delta u_{k}\right)$ which defines an asymptotically correct update strategy. Globalization strategies (line-search or trust region strategy) can also be applied.

The idea of the PRSQP methods formulated above can be pursued even further. For example, the constraint $c_{1}$ includes collocation conditions for the differential and for the algebraic variables and continuity conditions. If we conceive the nonlinear optimization problem (Eq. (3.7)) in a more generic sense, we may consider only the collocation conditions or even only the collocation conditions for the algebraic variables as the constraint $c_{1}$ and summarize the remaining discretization equations in $c_{2}$. Then, of course, the variables $u$ include more variables than just the control variables. Thus, a whole family of methods can be defined, differing in the degree of reduction, which includes (full) SQP methods and (fully) reduced SQP methods as special cases. It is also possible to pick variants that provide a special block structure in the reduced Hessian matrix, enabling the incorporation of the fast structured QP solvers developed in Steinbach (1995). Another important aspect especially with largescale applications for PDE models is the consistent treatment of inexact solutions of the linear subproblems. This topic in the context of PRSQP methods together with other practical aspects for discretized optimization problems is considered in Schulz (1997).

\section{Implementation issues}

For the numerical solution of the optimal control problems considered in this paper we couple the 


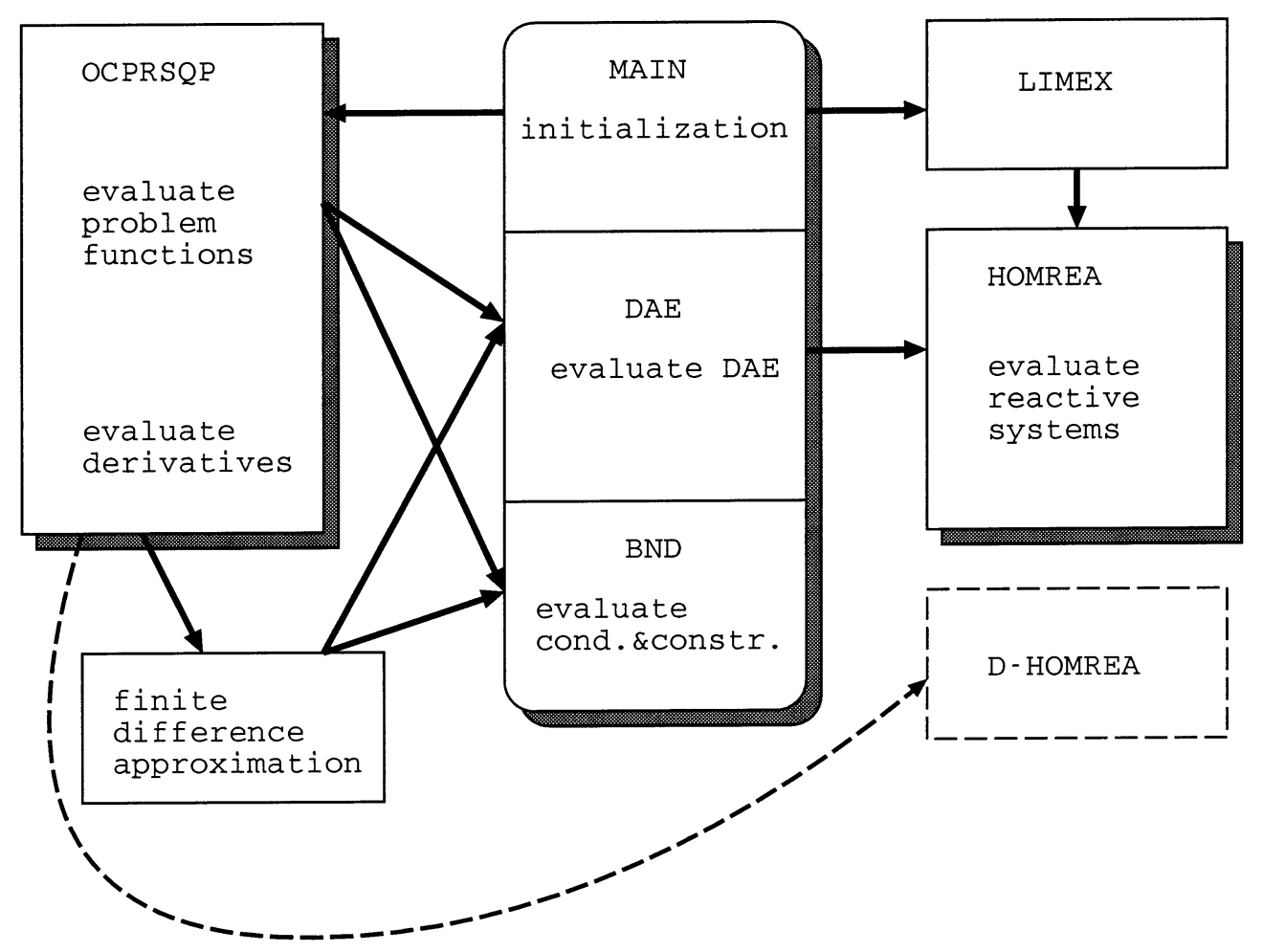

Fig. 2. Coupling of OCPRSQP and HOMREA.

optimal control problem solver OCPRSQP (Optimal Control problem solver employing a PRSQP method) and the package HOMREA used to simulate homogeneous reactive systems (Warnatz et al., 1996). Some details of this coupling are commented on in the following.

Those parts of the package HOMREA which exchange data with the optimization code compute the reaction rates of the gas phase species as a function of gas phase concentrations and temperature, i.e. the code provides the right hand side of equation (Eq. (2.1a)). Here, three subroutines of the package HOMREA are used. During the initialization of the problem the subroutine HORIN1 reads all chemistry specific data such as the stoichiometric matrix and the rate coefficients of the detailed reaction mechanism. Subroutine HORMEC calculates the reaction rates due to all elementary reactions.

OCPRSQP implements a PRSQP algorithm as described in the previous section. The variables $u$ in Eq. (3.7) are the discretized control variables together with the initial values of the differential variables and $y$ contains all remaining variables of the nonlinear program. Correspondingly, the constraints $c_{1}$ include the collocation and continuity conditions, whereas $c_{2}$ and $c_{3}$ contain the boundary conditions together with the control and state constraints.

The solution routine applied to the reduced quadratic programs of step 3 of the algorithm above is SOL/QPSOL (Gill, Murray, Saunders \& Wright, 1983) (in the NAG library Numerical Algorithm Group (1991) implementation E04NAF). The stopping criterion for the nonlinear iterations is defined by a user specified tolerance:

$\left|\frac{\mathrm{d}}{\mathrm{d} \tau} \Phi\left(x_{k}+\tau \Delta x_{k}\right)\right| \leq \mathrm{TOL}$,

where $x_{k}, \Delta x_{k}$ denote all variables and increments at the $k$ th PRSQP iteration and $\Phi$ the Powell exact merit function (Powell, 1978). This criterion measures a first order approximation of the decrease in the exact merit function to be expected from the increment $\Delta x_{k}$. Throughout all numerical studies in this paper, only one collocation interval is used per control interval.

Fig. 2 displays the user interface of OCPRSQP and the coupling with HOMREA in a schematic way. In order to interact with OCPRSQP, the user must provide a main program which allocates the necessary storage space and provides initial guesses for the variables. This main program calls OCPRSQP as a subroutine. Furthermore, the user has to provide a subroutine DAE that evaluates the right hand side of the model DAE, and a subroutine BNDS which evaluates the boundary conditions and state constraints. The latter two routines in turn are called OCPRSQP if an evaluation of the problem functions is necessary. 
The coupling with HOMREA is performed in two ways. On the one hand, it is called from an integration routine (here LIMEX (Deuflhard, Hairer \& Zugck, 1987)) in order to compute good initial guesses for the initial trajectory in the main program). On the other hand, it is called from the user provided routine DAE in order to evaluate the specific right hand side of the DAE for the reactive systems under consideration.

OCPRSQP necessitates not only the evaluation of the problem functions themselves but also of their derivatives. As a first approach, this is enabled in the current version by an intermediate routine implementing a finite difference approximation of the derivatives in order to make the coupling of OCPRSQP and HOMREA as loose as possible for testing purposes. Thus, prominent sparsity structures arising in the right hand side of the DAE are ignored, which accounts for some loss in the performance of OCPRSQP. The future implementation of a derivative routine D-HOMREA providing the sparsity structure of the system DAE should decrease the computing times considerably. This latter point is indicated in Fig. 2 by the dashed lines.

\section{Numerical results}

By using OCPRSQP we were able to solve the described optimal control problem for methane conversion to ethylene. 13315 variables occurred in the optimization problem resulting from 618 reactions and 55 differential equations in 61 control intervals. For this number of control intervals an error indicator as described in Schulz, 1996 indicates an error below $10^{-3}$ due to the discretization of the optimal control. We used three Radau points per collocation interval in order to obtain a stiffly stable discretization of high order. The algorithm took 50 iterations to converge to a precision of $\mathrm{TOL}=10^{-6}$, which required a cpu time of $39 \mathrm{~min}$ on a $400 \mathrm{MHz}$ PC with Pentium II processor.
The initial conditions at time $t=0$ correspond to a case given in the literature ( $\mathrm{Zhu}, 1993)$. The concentration of the species in $\mathrm{mol} / \mathrm{m}^{3}$ are $\mathrm{CH}_{4}: 12.56, \mathrm{O}_{2}: 5.15$, $\mathrm{H}_{2} \mathrm{O}: 0.913, \mathrm{CO}: 0.350, \mathrm{H}_{2}: 0.233, \mathrm{CO}_{2}: 0.117, \mathrm{C}_{2} \mathrm{H}_{4}$ : $0.06, \mathrm{C}_{2} \mathrm{H}_{6}: 0.06$; the pressure is 1.7 bar. Hence, the initial $\mathrm{CH}_{4} / \mathrm{O}_{2}$ ratio is 2.4 .

The initial profiles used for the optimization procedure are shown in the Figs. 3 and 4 on the left side. Here, a constant temperature of $1052 \mathrm{~K}$ is used. The maximum $\mathrm{C}_{2} \mathrm{H}_{4}$ concentration is $0.2 \mathrm{~mol} / \mathrm{m}^{3}$, achieved after a residence time of $0.4 \mathrm{~s}$ as shown in Fig. 3.

Optimization leads to an ideal reaction time of $t_{\text {end }}=$ $0.65 \mathrm{~ms}$ and yields an ethylene concentration of 0.5 $\mathrm{mol} / \mathrm{m}^{3}$, which is much higher than that achieved for the start profile.

The most interesting result is that the optimization yields a completely different residence time. Fig. 4 shows the concentration profiles of further important chemical species. After a reaction time of $0.65 \mathrm{~ms}$, the conversion of methane is $30 \%$, and the $\mathrm{C}$ atom selectivity for the desired $\mathrm{C}_{2} \mathrm{H}_{4}$ is $24.1 \%$. Most of the methane is converted into synthesis gas $\left(\mathrm{CO}\right.$ and $\left.\mathrm{H}_{2}\right)$ with a $\mathrm{CO}$ selectivity of $60 \%$. The other higher hydrocarbons formed are $\mathrm{C}_{2} \mathrm{H}_{6}(2.3 \%)$ and $\mathrm{C}_{2} \mathrm{H}_{2}(3.8 \%) . \mathrm{C}_{2} \mathrm{H}_{2}$ is directly formed from $\mathrm{C}_{2} \mathrm{H}_{4}$, which can only be stopped by a rapid temperature drop after $0.65 \mathrm{~ms}$. The very undesired complete oxidation products could even by decreased by the optimization procedure without directly focusing on that. The $\mathrm{CO}_{2}$ selectivity is $4 \%$.

The results of the optimization show that the maximum ethylene yield can be achieved if the residence time is very short $<1 \mathrm{~ms}$, and a rather high temperature is used. Actually, the ideal temperature is at the upper limit of $1600 \mathrm{~K}$, but only at a very short time, then the temperature must be decreased very fast to freeze the non-equilibrium product distribution. The oxygen conversion is only $60 \%$, which also indicates that a longer residence time would lead to a higher amount of complete oxidation products.
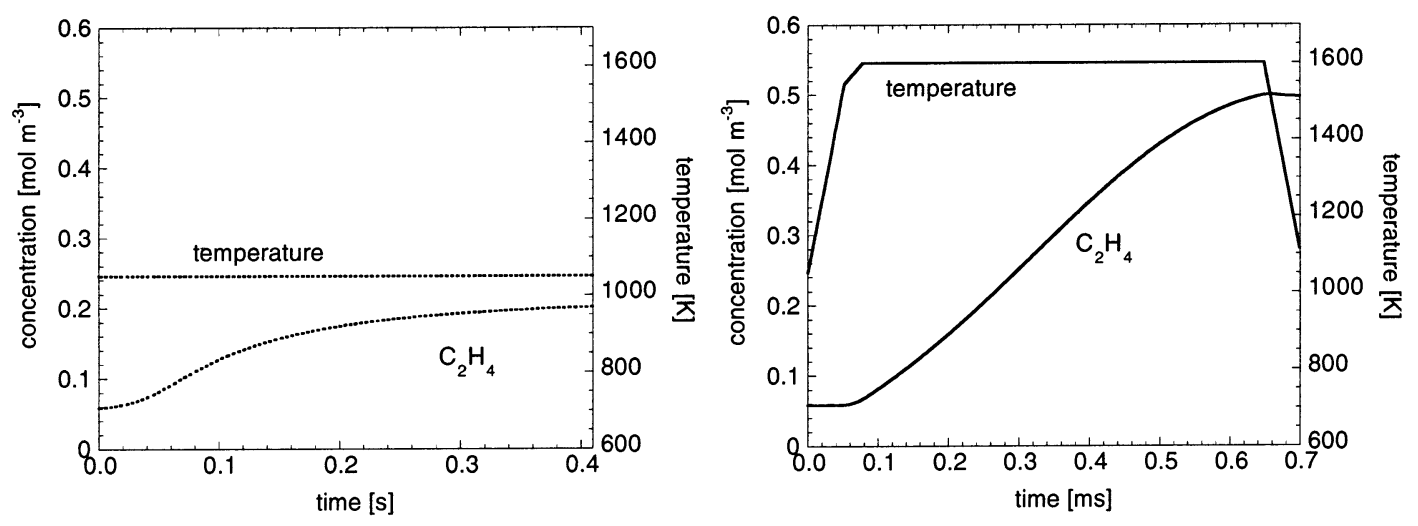

Fig. 3. Initial (left) and optimized (right) objective function $\left(\mathrm{C}_{2} \mathrm{H}_{4}\right)$ concentration and control (temperature) as a function of time; time scales are different. 

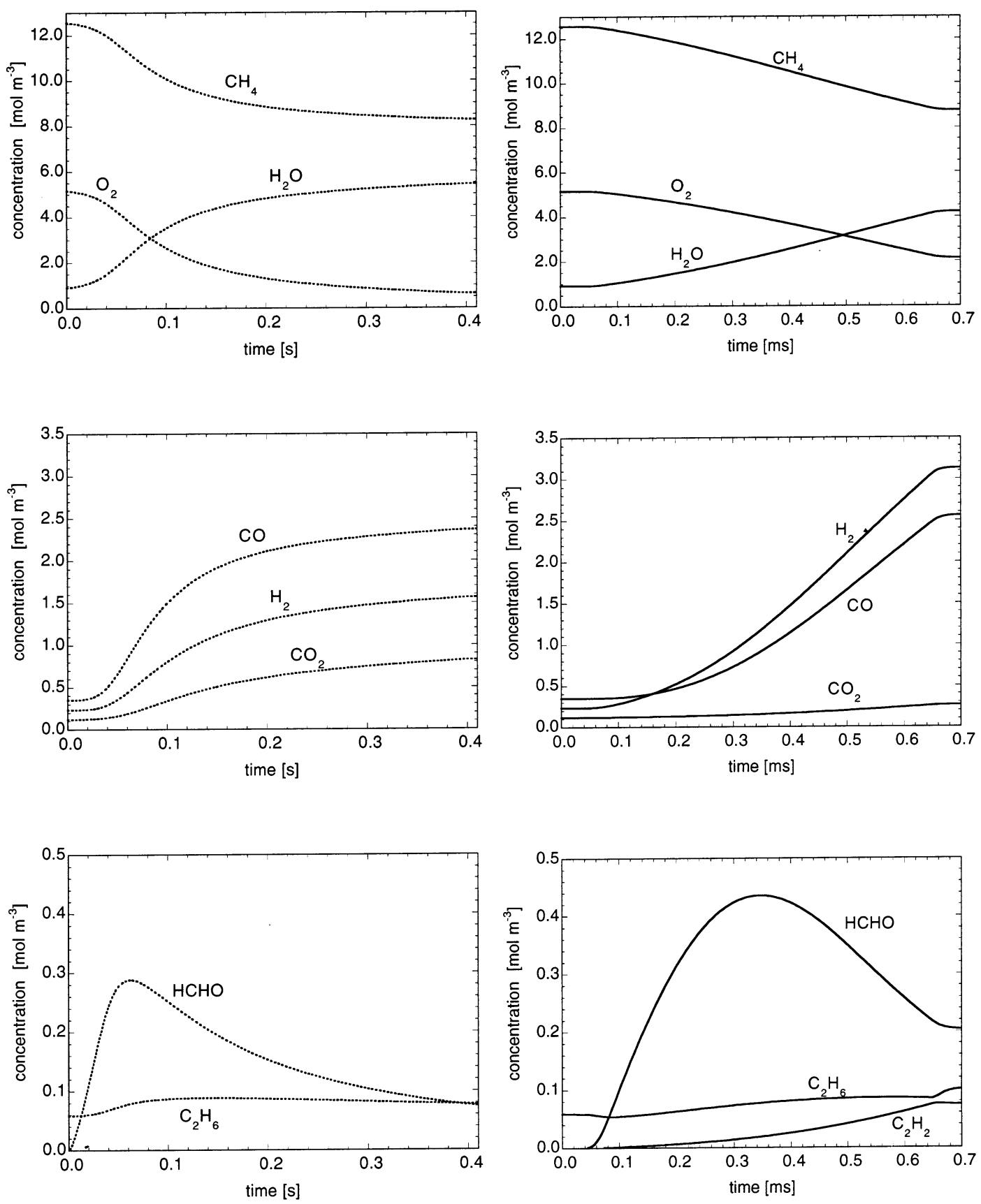

Fig. 4. Original and optimized species concentrations.

\section{Conclusions}

Efficient methods for the numerical computation of optimal controls for reactive systems are bound to become of similar importance for the competitiveness of chemical industry as are now pure numerical simulations of such processes. In the present paper we present a specific new optimal process control problem which is solved by a combination of a recently developed code for the numerical computation of optimal control problems with a code for simulation of the relevant dynamic processes. The combined code is shown to yield optimal control functions increasing the efficiency of the process under investigation. As it is pointed out in Section 4, there is potential for further algorithmic improvement of the combined method with respect to run-times, as well as to the accessible problem sizes. These further developments will take even more problem structure into account, and will thus enable the solution of even larger problems in less time. Furthermore, the coupling of the optimization code and a one-dimensional reactive flow code including detailed gas and surface chemistry is under development. 


\section{Acknowledgements}

The authors are indebted to H.G. Bock and J. Warnatz for many fruitful discussions and helpful advice during the preparation of this paper. In addition, they thank the Deutsche Forschungsgemeinschaft (DFG) for the support within the Sonderforschungsbereich 359 'Reaktive Strömungen, Diffusion and Transport'.

\section{References}

Ascher, U. M., \& Spiteri, R. J. (1994). Collocation software for boundary value differential-algebraic equations. SIAM Journal on Scientific Computing, 15(4), 938-952.

Baulch, D. L., Cobos, C. J., Cox, R. A., Esser, C., Frank, P., Just, Th., Kerr, J. A., Pilling, M. J., Troe, J., Walker, R. W., \& Warnatz, J. (1992). Evaluated kinetic data for combustion modelling. Journal of Physics and Chemistry Reference Data, 21, 411.

Biegler, L. T., Nocedal, J., \& Schmid, C. (1995). A reduced Hessian method for large-scale constrained optimization. SIAM Journal on Optimization, 5(2), 314-347.

Bock, H. G., \& Plitt, K. J. (1984). A multiple shooting algorithm for direct solution of constrained optimal control problems. In: Proceedings of the ninth IFAC world congress on automatic control. Pergamon Press.

Bock, H. G. (1987). Randwetproblemmethoden zur Parameteridentifizierung in Systemen nichtlinearer Differentialgleichungen, Bonner Mathematische Schriften 183, Bonn.

Cervantes, A., \& Biegler, L. T. (1998). Large scale DAE optimization using simultaneous nonlinear programming formulations. American Institute of Chemical Engineers Journal, 44(5), 1038.

Cervantes, A., \& Biegler, L. T. (2000). A stable elemental decomposition for dynamic process optimization, Journal of Computational and Applied Mathematics (in press)

Deuflhard, P., Hairer, E., \& Zugck, J. (1987). One-step and extrapolation methods for differential-algebraic systems. Numerical Mathematics, 51, 501-516.

Deutschmann, O. D., \& Schmidt, L. D. (1998). Modeling the partial oxidation of methane in a short-contact-time reactor. American Institute of Chemical Engineers Journal, 44, 2465-2476.

Gabay, D. (1982). Reduced quasi-Newton methods with feasibility improvement for nonlinear constrained optimization. Mathematical Programming Study, 16, 18-44.

Gill, P. E., Murray, W., Saunders, M. A., \& Wright, M. H. (1983). User's guide for SOL/QPSOL. Technical Report SOL 83-7, Department of Operations Research, Standford University, Standford, CA.

Gurwitz, C. B., \& Overton, M. L. (1989). Sequential quadratic programming methods based on approximating a projected Hessain matrix. SIAM Journal on Scientific and Statistical Computing, 10(4), 631-635.

Hickman, D. A., \& Schmidt, L. D. (1993). Steps in CH4 oxidation on Pt and Rh surfaces: High-temperature reactor simulations. American Institute of Chemical Engineers Journal, 39, 1164-1177.
Kupfer, F.-S., \& Sachs, E. W. (1993). Reduced SQP methods for nonlinear heat conduction control problems. International Series of Numerical Mathematics, 111, 145-160.

Lane, G. S., \& Wolf, E. E. (1988). Methane utilization by oxidative coupling. Journal of Catalysis, 113, 144-163.

Leineweber, D. B., Bock, H. G., Schlöder, J. P., Gallitzendörfer, J. V., Schäfer, A., \& Jansohn, P. (1997). A boundary value problem approach to the optimization of chemical processes described by DAE models. Technical Report Preprint 97-14, IWR, University of Heidelberg.

Nehse, M. (1999). Automatische Erstellung von detaillierten Reaktionsmechanismen zur Modellierung der Selbstzündung und laminarer Vormischflammen von gasförmigen KohblenwasserstoffMischungen. PhD thesis, Universität Heidelberg.

Nocedal, J., \& Overton, M. L. (1985). Projected Hessain updating algorithms for nonlinearly constrained optimization. SIAM Journal on Numerical Analysis, 22(5), 821-850.

Numerical Algorithm Group (1991). The NAG FORTRAN library manual, mark 15, Oxford.

Powell, M. J. D. (1977). A fast algorithm for nonlinearly constrained optimization calculations. In G. A. Watson, Numerical Analysis Proceedings Dundee 1977 (pp. 144-157). Springer-Verlag.

Schulz, V., \& Deutschmann, O. (1999). Process optimization of reactive systems modeled by elementary reactions. In F. Keil, W. Mackens, H. Voss, \& J. Werther, Scientific Computing in Chemical Engineering II (pp. 354-361). Springer.

Schulz, V. H. (1996). Reduced SQP methods for large-scale optimal control problems in DAE with application to path planning problems for satellite mounted robots. PhD thesis, University of Heidelberg.

Schulz, V. H. (1997). Solving discretized opmization problems by partially reduced SQP methods. Computer Visual Science, 1, 2.

Schulz, V. H., Bock, H. G., \& Steinbach, M. C. (1998). Exploiting invariants in the numerical solution of multipoint boundary value problems in DAE. SIAM Journal on Scientific Computing, 19, 2.

Steinbach, M. C. (1995). Fast recursive SQP methods for large-scale optimal control problems. PhD thesis, University of Heidelberg.

von Schwerin, M. (1997). Numerische Methoden zur Schätzung von Reaktions-geschwindigkeiten bei der katalytischen Methankonversion and Optimierung von Essigsäure- und Methanprozessen. PhD thesis, Universität Heidelberg.

von Schwerin, R., Winckler, M., \& Schulz, V. (1996). Parameter estimation in discontinuous descriptor models. In D. Bestle, \& W. Schiehlen, IUTAM Symposium on Optimization of Mechanical Systems (pp. 269-276). Kluwer Academic Publishers.

Warnatz, J. (1992). Resolution of gas phase and surface combustion chemistry into elementary reactions. In: The twenty-fourth symposium (international) on combustion (pp. 553-579). The Combustion Institute.

Warnatz, J., Dibble, R. W., \& Maas, U. (1996). Combustion, physical and chemical fundamentals, modeling and simulation, experiments, pollutant formation. New York: Springer-Verlag.

Zhu (1993). Application of sensitivity analysis to the reduction of a complex kinetic model for the homogeneous oxidative coupling of methane. Chemical Engineering and Processing, 32, 167. 